\title{
ASSESSING THE CONDITION OF BURIED PIPE USING GROUND PENETRATING RADAR (GPR)
}

\author{
Saiful Wazlan Wahab ${ }^{1,3}$, D.N. Chapman ${ }^{1}$, C.D.F. Rogers ${ }^{1}$, K.Y. Foo ${ }^{2}$, N. Metje ${ }^{1}$, Nawawi S.W. ${ }^{4}$, Mohd Noor Isa ${ }^{3}$, Madun A. \\ ${ }^{1}$ School of Civil Engineering, University of Birmingham, Edgbaston, Birmingham B15 2TT, United Kingdom \\ ${ }^{2}$ Pulsar Process Measurement Ltd., Cardinal Building, Enigma Commercial Centre, Sandy's Road, Malvern, Worcestershire, WR14 \\ 1JJ, United Kingdom. \\ ${ }^{3}$ Department of Survey and Mapping Malaysia, Wisma JUPEM, Jalan Sultan Yahya Petra, 50578 Kuala Lumpur, Malaysia \\ ${ }^{4}$ Universiti Teknologi Malaysia, 81310 Skudai, Johor, Malaysia \\ ${ }^{5}$ Universiti Tun Hussein Onn Malaysia, 86400 Parit Raja, Johor, Malaysia \\ *Email: saiful@jupem.gov.my
}

KEY WORDS: Ground Penetrating Radar (GPR), Pipe deterioration, Buried utilities

\begin{abstract}
:
The invention of Ground Penetrating Radar (GPR) technology has facilitated the possibility of detecting buried utilities and has been used primarily in civil engineering for detecting structural defects, such as voids and cavities in road pavements, slabs and bridge decks, but has not been used to assess the condition of buried pipes. Pipe deterioration can be defined as pipes where, for example, cracking, differential deflection, missing bricks, collapses, holes, fractures and corrosion exists. Assessing the deterioration of underground pipes is important for service efficiency and asset management. This paper describes a research project that focused on the use of GPR for assessing the condition of buried pipes. The research involved the construction of a suitable GPR test facility in the laboratory to conduct controlled testing in a dry sand. Plastic pipes were chosen for the experiments. A series of laboratory experiments were conducted to determine the validity and effectiveness of standard commercially available GPR technology in assessing the condition of buried utilities with common types of damage. Several types of damage to the plastic pipe were investigated with respect to different GPR antenna frequencies. The GPR surveys were carried out in order to obtain signal signatures from damaged and undamaged pipes buried at $0.5 \mathrm{~m}$ depth. These surveys were organised on a grid pattern across the surface of the sand in the test facility. The results presented in this paper show that GPR can identify certain types of damage associated with a buried pipe under these controlled laboratory conditions.
\end{abstract}

\section{INTRODUCTION}

In the UK, most of the essential public service infrastructure was installed in the last two hundred years [1] which obviously the quality of buried pipes have become degraded. To overcome this issue, approximately four million holes were dug every year in UK roads in order to repair assets and new installations [2]. However, inaccurate asset management can lead to unnecessary holes dug in the wrong place and this often causes third party damage to other underground services. Those damages are potentially to lengthy delays to construction works and increasing the direct costs of maintenance to the service provider and not least to the social disruption as well. It is therefore important to accurately locate these services which could minimize unnecessary holes dug for repairing and maintenance of existing assets.

The invention of Ground Penetrating Radar (GPR) technology has facilitated the possibility of detecting buried utilities and has been used primarily in civil engineering for detecting structural defects, such as voids and cavities in road pavements, slabs and bridge decks[3], but has not been used to assess the condition of buried pipes. Pipe degraded can be defined as pipes where, for example, cracking, differential deflection, missing bricks, collapses, holes, fractures and corrosion exists[3,4,5].This GPR technology has become favorable geophysical technique in detecting underground utilities. However, this technique still has certain limitations which obviously depending on ground conditions, type and depth of objects. This feasibility research focuses on investigating whether off-the-shelf Ground
Penetrating Radar (GPR) can be used for assessing the condition of pipes.

\section{BASIC PRINCIPLES OF GPR}

GPR is a device used for non-invasive scanning which able to record accurate depth reading and the signature of targets (radagram) for further properties interpretations and mainly to detect shallow or deep targets depending on frequency of antenna is applied. The function of GPR wave transmission and reflection is shown in Figure 1.

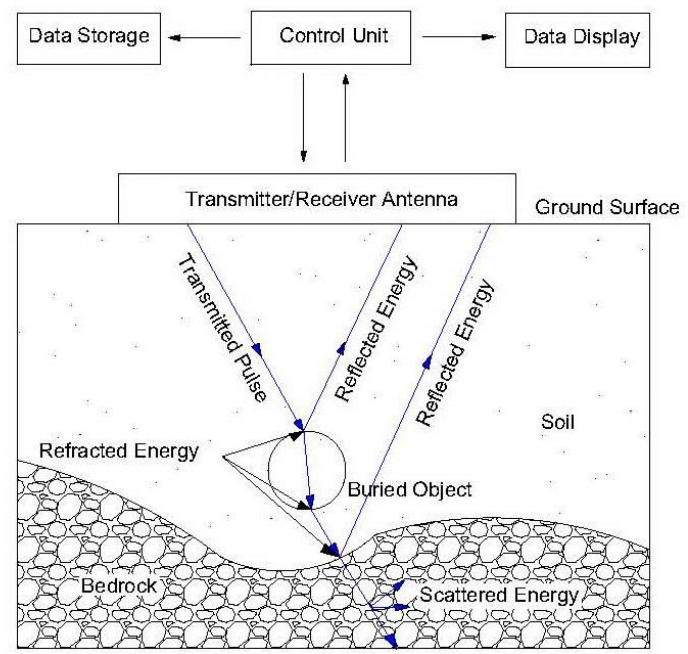

Figure 1: Functional diagram of GPR equipment [8] 
In physical brief, GPR is composed of a receiver and transmitter antenna, a control unit, battery supply and a survey cart. The control unit is the main part of the GPR because it controls the whole system. Generally, when the survey cart moves on the site surface the transmitting antenna sends polarized, high frequency electromagnetic (EM) waves into the ground. Because of different existing inhomogenities in the ground, e.g. soil layers, underground utilities, stones, gravel, cavities and other anomalies, a proportion of the EM waves are reflected from the dielectric boundary between different materials and the rest is refracted and continues to deeper layers. The process is repeated until the EM waves become too weak. Reflection of the EM waves from the dielectric boundary is the consequence of differences in the electric and magnetic properties of materials of infrastructural objects and soil layers [6].

As the conclusion, the GPR works by sending pulses of energy into the ground and recording the strength and the time for the return of any reflected signal. These series of pulses over a single area is called a scan. Reflection is occurred whenever the energy pulses are transmitted through various materials on their way to the buried target feature thus changes the velocity. Their velocity changes depending basically on two primary electrical properties of the subsurface: electrical conductivity $(\sigma)$ and dielectric permittivity $(\varepsilon)$. Reflections are produced by changes in the dielectric contrast due to changes in the subsurface materials [7]. The greater the contrast between two materials at subsurface interface, the stronger the reflected signal, resulting in a higher amplitude reflected wave [8].Therefore, it is necessary to understand those characteristics of the materials which affect both the velocity of propagation and attenuation.

\section{RESEARCH METHODOLOGY}

Even though the applications of GPR have been widely applicable, it was not much work on GPR for condition assessment. As the GPR has the limitations, approaching some controlled experiment in the laboratory is more reliable. In this research, a laboratory test facility consisting of a box of dimensions $2.4 \mathrm{~m}$ (length) $\times 2.2 \mathrm{~m}$ (width) $\times 1.2 \mathrm{~m}$ (height) constructed from structural insulated panel material is used. The size of the box was determined as the minimum requirement for the GPR unit based on beam width antenna calculations so as to avoid signal reflection from the edges or base of the box and to ensure the complete shape of the hyperbolic trace from the targets to be captured. For initial experiments, only a plastic pipe was chosen as the main pipe material. The plastic pipe was chosen in order to create simple pipe damage. The experiments involve burying $0.2 \mathrm{~m}$ diameter plastic pipes in the box (Figure 2). One of these pipes is in a 'good' state (undamaged) and the other has a defect (i.e. break or hole), i.e. damaged pipe.

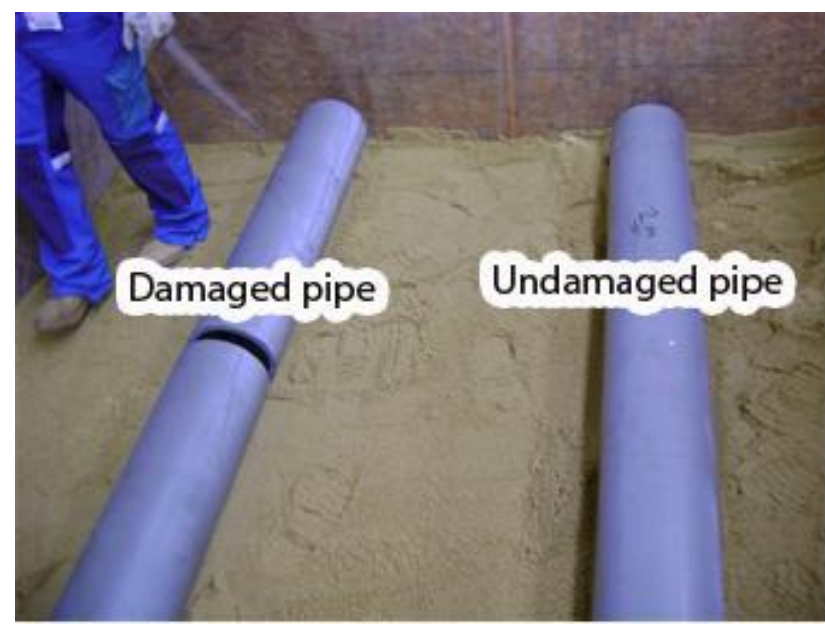

Figure 2: Arrangement of the two plastic pipes in the test box prior to burial

In these experiments, the depth of buried pipe was at $0.5 \mathrm{~m}$ $( \pm 0.02 \mathrm{~m})$ from the soil surface. Meanwhile, the position of buried pipe was laid at $1 \mathrm{~m}$ gap between each other and $0.4 \mathrm{~m}$ from the wall. This depth and position was chosen as an ideal orientation which could minimise those signals that are unrelated to the target (clutter). Clutter can be caused by breakthrough between the transmit and receive antennas as well as multiple reflections between the antenna and the ground surface[6]. Generally, clutter is more significant at short range times (shallow target) and decreases at longer times (deep target). In theory, in order to get the complete shape of hyperbola from the targets (Figure 3), Equation 1 had been applied which the width of the test facility must be at least $4 y$ i.e. $X=4 y$ :

$\mathrm{y}=\tan ^{-1} \theta(0.5)$

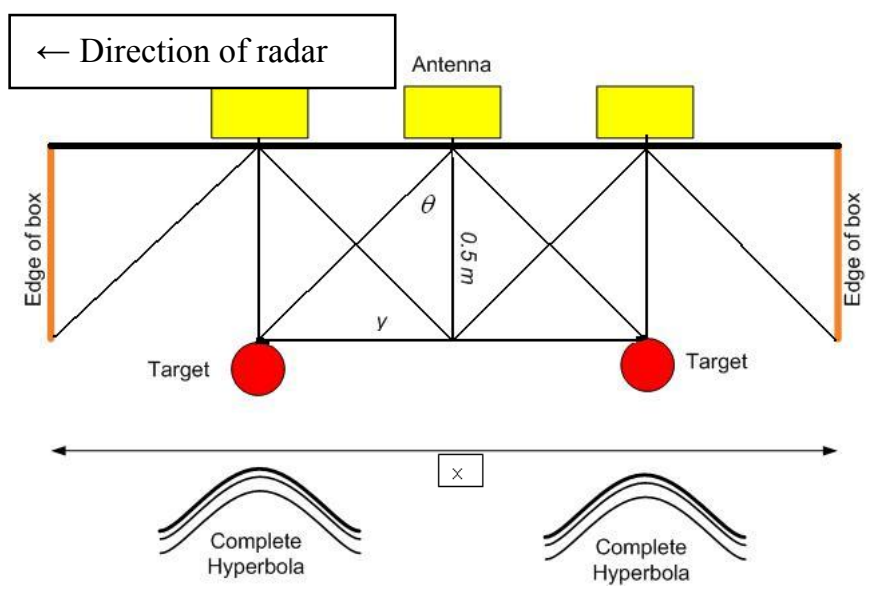

Figure 3: Minimum width of test facility

A schematic model of the test facility indicates the position of two pipes and was constructed as shown in Figure 4: 


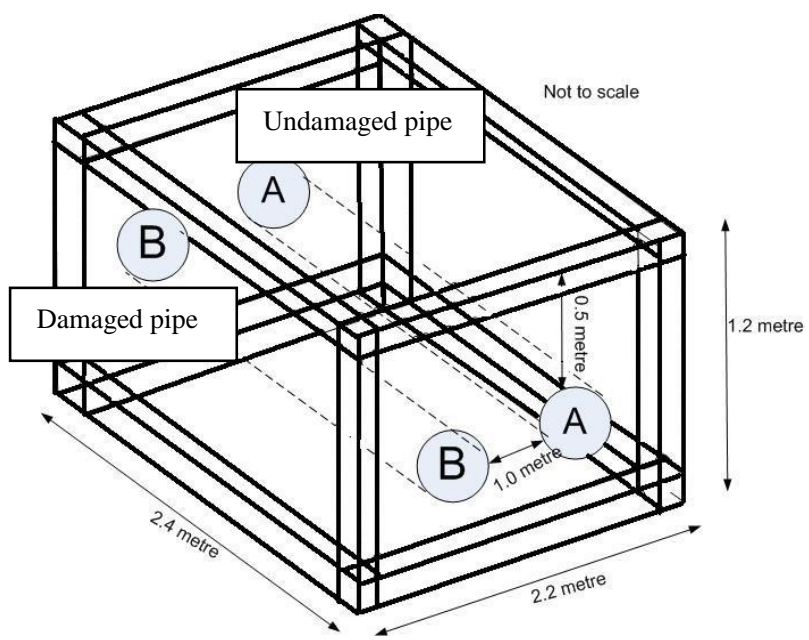

Figure 4: A schematic model of the test facility indicating the pipe position

In theory, the strength of electromagnetic waves depends on the medium they are passing through. The GPR signal can travel further in 'low-electrical-loss' materials or in other words propagation velocity increases with decreasing relative permittivity. The GPR signal would penetrate great deeper if the electrical conductivity is equal to zero [9]. Therefore, soil characteristic such as low-electrical-loss-materials, low relative dielectric constant and low absorption coefficient are needed as fill materials for test facility [10].

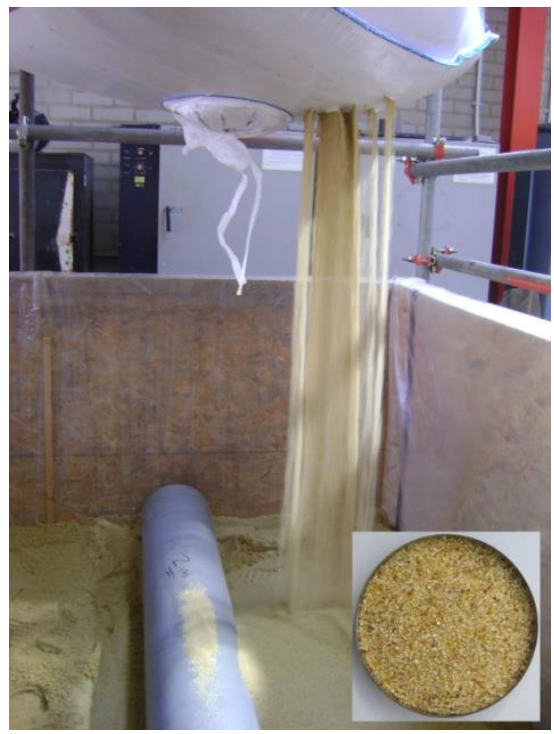

Figure 5: Fill material for test facility

In these experiments, a Leighton Buzzard sand is selected as the soil medium as shown in Figure 5. This soil medium is selected as represent the 'best' condition in order to observe pipe condition in these initial tests (if it was not possible to see any differences in the pipe using the GPR under this condition then it would not be worth doing further experiments with more 'realistic' soil).

A series of laboratory experiments were conducted to determine the validity and effectiveness of the GPR technology in assessing the condition of buried utilities with common plastic pipe damages. Several damages in plastic pipe were tested with respect to different GPR antenna frequencies e.g $250 \mathrm{MHz}$ and $700 \mathrm{MHz}$. The GPR surveys were carried out in order to obtain signal signatures from damaged and undamaged pipes. These surveys were organised through grid pattern across a test facility (Figure 6).

$0.1 \mathrm{~m}$ intervals

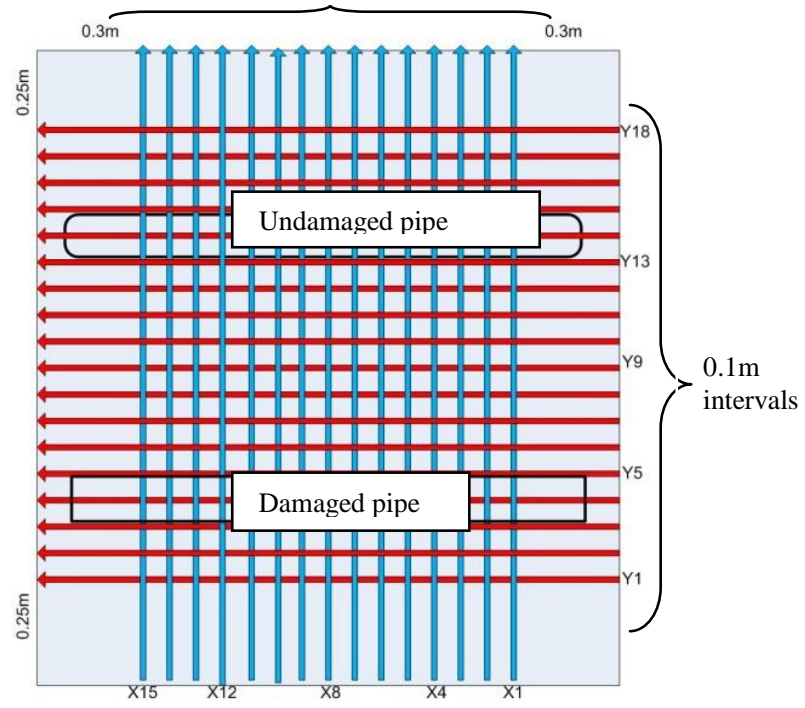

Figure 6: Survey grid lines used for each experiment

In practice, the interval spacing of the GPR survey grid should be varied depending on the purpose of the survey require, i.e. larger for location, and smaller for utility assessment. For this research a number of grid spacing were tried and based on the finding, a $0.1 \mathrm{~m}$ spaced GPR survey grid was found to be appropriate for the current experiments in both the direction of the pipes ( $\mathrm{Y}$ direction) and perpendicular to the pipes (X direction)

During the experiments, the IDS K2-Fastwave software was used to capture the data. Ultimately, the information of all the radar signals were processed, extracted and were further examined and analyzed.

\section{RESULTS AND DISCUSSION}

Soils are generally considered non-magnetic materials hence the permittivity and conductivity are the two most important parameters that influence the signal velocity and attenuation [10]. Therefore the relative dielectric permittivity and conductivity of the sand was determined as $\varepsilon_{\mathrm{r}}=2.72$ and $\sigma=$ $0.01 \mathrm{SM}^{-1}$, respectively by using Time Domain Reflectometry (TDR). From this result, the velocity of the signal was calculated as $v=180 \mathrm{~mm} / \mathrm{ns}$ for use in the analysis of the GPR data. This value can be compared with the velocity value as stated in Table 2 and it is acceptable.

\begin{tabular}{|l|l|l|}
\hline Materials & $\begin{array}{c}\text { Relative permittivity } \\
\left(\varepsilon_{\mathrm{r}}\right)\end{array}$ & Velocity $(\mathrm{mm} / \mathrm{ns})$ \\
\hline Dry Sand & $2-6$ & $120-170$ \\
\hline Wet Sand & $10-30$ & $55-60$ \\
\hline
\end{tabular}

Table 2: Dielectric value for common materials [6]

For initial test, the GPR was ran perpendicular to the pipes and an example of the signal contrast between a damaged pipe and an undamaged pipe when the GPR crosses perpendicular to the pipe direction is shown in Figure 7. 


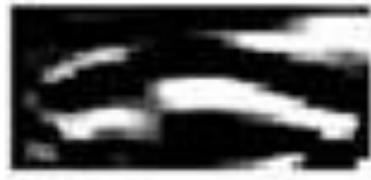

(a)

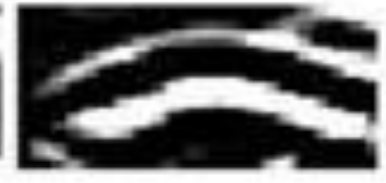

(b)
Figure 7: Signal contrast between (a) a damaged pipe and (b) an undamaged pipe.

The $0.1 \mathrm{~m}$ survey grid produces 18 points along and 15 crossing points perpendicular to the pipe. Fourteen tests were conducted as part of this research. These tests are 1) broken pipe split into two sections with a $5 \mathrm{~cm}$ gap without plastic cover 2) broken pipe with a $5 \mathrm{~cm} \& 2 \mathrm{~cm}$ gap with plastic cover 3 ) hole in the pipe with a diameter of $5 \mathrm{~cm} \mathrm{4)} \mathrm{hole} \mathrm{in} \mathrm{the} \mathrm{pipe} \mathrm{with} \mathrm{a} \mathrm{diameter} \mathrm{of}$ $5 \mathrm{~cm}$ covered by polystyrene 5) hole in the pipe with a diameter of $5 \mathrm{~cm}$ covered by fabric 6 ) broken pipe with $5 \mathrm{~cm}$ gap (sand prevented from passing through the gap by a fabric cover) 7) a hole in the pipe with a diameter of $5 \mathrm{~cm}$ (a sponge was used to cover inside the pipe).

All the signal signatures captured by the GPR were identified and analysed. Commercial IDS software was used to capture and process the data. Meanwhile, the data analysis was based on the signal contrast between the two types of pipe (damaged and undamaged). Advanced interpretation (Matlab programming) was used to differentiate the signal amplitude between the different pipes using a Mean Square Error (MSE) analysis. The analysis focussed on the amplitude changes of particular areas of the GPR data obtained from the undamaged and damaged pipe. In order to verify consistency of the data, three sets of GPR data were taken for each test and averaged after MSE analysis had been done (after confirming that they were similar). From these experiments, interpretation through radar images is quite hard to identify any damages to the pipe. However, this drawback can be solved through intelligence Matlab programming but only in certain conditions and these are still limitations.

\section{CONCLUSION}

This work has successfully shows both frequencies of $250 \mathrm{MHz}$ and $700 \mathrm{MHz}$ are capable to observe the defective regions, but only in certain conditions and these are still limitations. Although both frequencies were capable of observing the defective region, the antenna with $250 \mathrm{MHz}$ frequency has some drawbacks. The radar scan from the $250 \mathrm{MHz}$ antenna was blurred and darker due to reduced signal resolution. In addition, $250 \mathrm{MHz}$ antenna produced less returning signal compared to the $700 \mathrm{MHz}$ antenna. This is because the wavelength of 250 $\mathrm{MHz}$ is much longer compared to $700 \mathrm{MHz}$. However, the 700 $\mathrm{MHz}$ antenna shared more signal attenuation compared to 250 $\mathrm{MHz}$. Any signal interference from the surroundings such as from radio waves, cellular radio, television, satellite radio and microwaves are factors affecting the radar scan results.

In these experiments, several types of damaged pipe were investigated. The damaged pipe involved broken sections and hole in the pipe. Both type of damage were tested under different conditions. Firstly, allowing the soil to pass through the damaged region and secondly where the soils is prevented from entering the damaged region. Radar scans were conducted in both direction e.g perpendicular to the pipes and along the pipes. Two results were identified; Firstly, conducting the radar scan perpendicular to the pipe had a better result compared to scanning along the pipes. It was quite hard to interpret the radar scan along the pipe because it just showed one thick straight line and it was very hard to identify any changes in the images and thus hard to quantify and identify the amplitude changes in the damaged region.

Secondly with respect to the damaged on the pipe, the vertically broken pipe was easier to identify and to quantify compared to the hole in the pipe. The reason for this involved the amount of soil entering the pipe via the damaged region. More soil in the pipe made for easier interpretation and clarification. However, the vertical break in the pipe and the hole in pipe couldn't be identified and detected if the soil was prevented from passing through the damaged regions. The amount of soil passing through the damaged region is a key factor in identifying the damaging region. The more soils materials passing through, the better the results and clearer the radar image that is achieved.

All the objectives of the research have been carefully undertaken to achieve the initial aim. By considering to the outputs from the analyses and the objectives of this research, it can be concluded that:

i) Different antenna frequencies will results in different signal signatures between undamaged and damaged pipes in terms of image resolutions and signal attenuation (clutter);

ii) GPR, with careful analysis of the signals, can identify damage in pipes under the controlled conditions in these experiment but still has limitations. This limitations depend on the size of damaged region and the type of damage. However, the damage was easily identified if it was associated with other materials (soil). Without these materials, it was hard to identify the damage;

iii) The GPR signal of damaged regions relative to undamaged regions under 'ideal' ground condition are depends on the amount of soils (materials) passing through the damaged regions. The more soil passing into the pipe will give a better result in term of identifying the damaged region.

\section{ACKNOWLEDGEMENT}

The authors would like to express sincere appreciation and special thanks to Principal Investigator of Mapping the Underworld, University of Birmingham, UK. The research program was funded from Engineering and Physical Sciences Research Council (EPSRC). Also sincere thanks to Department of Survey and Mapping Malaysia for their support.

\section{REFERENCES}

[1] Metje, N., Chapman, D.N., Rogers, C.D.F., and K.Bongs (2011). Seeing through the ground: The potential of gravity gradient as a complementary technology. Journal of Advances in Civil Engineering, Vol. 2011, Article ID 903758, 9 pages, 2011.

[2] Beck, A.R., Fu, G.,Cohn, A.G., Bennett, B., and Stell, J.G., 2008. A framework for utility data integration in the UK. In: V. Coors, M. Rumor, E.M. Fendel and S. Zlatanova (Editors), Urban and Regional Data Management - Proceeding of the Urban Data Management Society Symposium 2007 Taylor and Francis, London, pp. 261-276 
[3] Koo, D. H., Ariaratnam, S. T. (2006). Innovative method for assessment of underground sewer pipe condition. Journal of Automation in Construction, 15, 479-488.

[4] Sinha, S. K., \& Knight, M. A. (2004). Intelligent System for Condition Monitoring of Underground Pipelines. ComputerAided Civil and Infrastructure Engineering, 19, 42-53.

[5] Silva, D. D., Davis, P., Burn, L. S., Ferguson, P., Massie, D., Cull, J., Eiswirth, M., Heskee, C. (2002). Condition Assessment of Cast Iron and Asbestos Cement Pipes by In-Pipe Probes and Selective Sampling for Estimation of Remaining Life. No Dig. Australia.

[6] Daniels, D. J. (2004). Ground Penetrating Radar . London: The Institution of Engineering and Technology,2nd Edition, 227.

[7] Eyuboglu, S., Mahdi, H., Al-shukri, H., and Rock, L. (2003). Detection of water leaks using Ground Penetrating Radar.

[8] Kuo, S., Zhoa, L., Mahgoub, H., and Suarez, P. (2005). Investigation on ground penetrating radar for detection leaking pipelines under roadway pavements and development of fiber wrapping repair technique. Transportation.US

[9] Jol, H., (2009). Ground Penetrating Radar: Theory and Applications (pp. 41-54). Oxford: Elsevier Science. 1-143

[10] Royal, A.C.D., Rogers, C.D.F., Atkins, P.R., Chapman, D.N., Curioni, G., Foo, K.Y., Hao, T., Metje, N., Moghareh Adeb, T., Shirgiri, N., and Wazlan, S. (2011). Pipeline Engineering in the ground: The impact of ground conditions on pipeline condition and maintenance operations. International Conference on Pipelines and Trenchless Technology (ICPTT), 2011. Beijing, China., 1598-1609.

Revised August 2018 